\title{
From dynamics to links: a sparse reconstruction of the topology of a neural network
}

\author{
Giacomo Aletti ${ }^{1}$, Davide Lonardoni ${ }^{2}$, Giovanni Naldi ${ }^{1}$, Thierry Nieus ${ }^{3,2 *}$ \\ ${ }^{1}$ ADAMSS Center, Università degli Studi di Milano, 20131 Milano, Italy \\ ${ }^{2}$ Neuroscience Brain Technology, Istituto Italiano di Tecnologia, via Morego 30, 16163 Genova, Italy \\ ${ }^{3}$ Dipartimento di Scienze Biomediche e Cliniche 'Luigi Sacco', Università degli Studi di Milano, \\ 20157 Milano, Italy \\ *Email address for correspondence: thierry.nieus@unimi.it \\ Communicated by Michele Piana and Luigi Preziosi \\ Received on 11 29, 2016. Accepted on 11 13, 2017.
}

\begin{abstract}
One major challenge in neuroscience is the identification of interrelations between signals reflecting neural activity and how information processing occurs in the neural circuits. At the cellular and molecular level, mechanisms of signal transduction have been studied intensively and a better knowledge and understanding of some basic processes of information handling by neurons has been achieved. In contrast, little is known about the organization and function of complex neuronal networks. Experimental methods are now available to simultaneously monitor electrical activity of a large number of neurons in real time. Then, the qualitative and quantitative analysis of the spiking activity of individual neurons is a very valuable tool for the study of the dynamics and architecture of the neural networks. Such activity is not due to the sole intrinsic properties of the individual neural cells but it is mostly the consequence of the direct influence of other neurons. The deduction of the effective connectivity between neurons, whose experimental spike trains are observed, is of crucial importance in neuroscience: first for the correct interpretation of the electro-physiological activity of the involved neurons and neural networks, and, for correctly relating the electrophysiological activity to the functional tasks accomplished by the network. In this work, we propose a novel method for the identification of connectivity of neural networks using recorded voltages. Our approach is based on the assumption that the network has a topology with sparse connections. After a brief description of our method, we will report the performances and compare it to the cross-correlation computed on the spike trains, which represents a gold standard method in the field.
\end{abstract}

Keywords: Neural Networks; Sparse reconstruction; LASSO method

AMS subject classification: 92B20; 65K05; 37F99

\section{Introduction}

Along the latest years, there has been enormous progress in the Neuroscience field that has revolutionized the way we envisage the brain functions. In this regard, the technological advancements have been fundamental to improve the recording capability from brain areas and neural populations [1]. Nowadays, multi-site recordings can be achieved from thousands of channels (sites) with a good spatial (at the cellular level) and temporal resolution (less than one millisecond for the action potential) yielding a good description of the underlying network dynamics. Given that the brain operates on a single trial basis such recordings are instrumental in understanding the neural code [2]. As a first step, multi-site recordings allow quantifying the information flow in the network. Although the anatomical wiring (i.e. Structural Connectivity, SC) can not explain the spiking activity by itself, it does play a fundamental role in limiting and regulating how cells interact with each other. However, even though SC might provide a valuable piece of information to understand how neuronal processing occurs, it is often not directly measurable. Consequently, other quantities, such as Functional Connections and Effective Connections, have been proposed to describe the relations among neurons in a network. For instance, multi-site recordings can be used to infer statistical dependencies (i.e. Functional Connections, FC) among the recorded units and to track the information flow in the network [3]. On the other hand, the Effective Connectivity (EC) denotes the directed causal relationship between the recorded sites. The EC is typically estimated, in vitro 
experiments, by stimulating one cell and studying the effects on the connected elements. Alternatively, the EC can also be studied establishing a causal mathematical model between the recorded units data. A powerful approach to estimate the EC of a set of neurons has to overcome some fundamental constraints that need to be carefully considered. First, in the worst-case scenario, the number of possible connections among neurons scales quadratically with the number of neurons. Second, experimental limitations set constraints on the duration of the recordings, i.e. on the information available. Third, the large number of simultaneously recorded units poses a set of numerical and mathematical problems that would be hard to face, in particular for long lasting recording sessions. These issues are common to different fields and have been coined as 'curse of dimensionality'. Two approaches are typically foreseen to overcome these points. A first solution consists in a reductionist approach that projects the data onto a lower dimensional space that can better elucidate the underlying processing. Another possibility consists into fitting the observed data to a low-dimensional model that captures the salient properties of the dynamics [1]. Here we present a model of effective connectivity that introduces a natural constraint of almost all biological networks to significantly reduce the dimensionality of the problem: the sparseness among the connected units (see e.g. [4]). Other approaches, such as the multivariate autoregressive model [5], allow for the possibility of a fully connected network in which every node may influence all the other nodes. However, this is somewhat unrealistic for biological networks (i.e. each neuron is directly influenced by only a small subset of neurons) and it also leads to practical challenges. In fact, most of the connectivity tools used to understand the communication among neuronal populations are based on linear models although it is widely recognized that the interactions (i.e. synaptic currents) among neural cells are nonlinear. Then, fully connected networks involve models that can easily be over-parameterized and hard to solve. Therefore current methods are not well suited to robustly infer on the network connectivity from the time series in different contexts. Among these approaches, the Granger causality $[6,7]$ is probably the most prominent and most widely used concept. Granger causality is a statistical method for assessing directional influences between simultaneously recorded time series. Recent work demonstrates that, when applied to electrophysiological data, the directions and magnitudes of Granger causality are interpretable in terms of the directions and magnitudes of synaptic transmissions between different neurons and brain areas $[7,8]$. For two simultaneously measured time series, if the variance of the prediction error for the second time series at the present time is reduced by including past measurements from the first time series in the linear regression model, then the first time series can be said to cause the second time series. This concept of causality does not rely on the specification of a scientific model and thus is particularly suited for empirical investigations of cause-effect relationships. On the other hand, if important relevant variables are not included in the analysis, the Granger causality can lead to so-called spurious causalities. Importantly, Granger causality based methods can be summed up as a theoretical framework based on conditional independence to assess directional dependencies between these time series. In order to capture nonlinear interactions between even short and noisy time series, we consider an event-based model. Then, we involve the physiological basis of the signal, which is likely non-linear. We point out that most connectivity methods usually rely on the timestamps of the action potentials (or spikes) as the sole source of information for inferring the effective connectivity. In this context, spikes of a given neuron are detected by applying a threshold on the raw intracellular or extracellular voltage trace discarding the sub-threshold activity $[9,10]$. Instead, in the approach introduced in this work, we suppose we can record the membrane potential of a large subset of neurons in a network or, more in general, to acquire information on the sub-threshold dynamics related to the integration of synaptic events. Differently from spike-time based methods, here we exploit both supra and sub-threshold events (spikes and synaptic activations respectively) to estimate the effective connectivity of a given network. Notably, similar data can be nowadays obtained from in vitro experiments [11] and, very recently, also in vivo with Optogenetics [12]. Consequently, this work sets the ground for the exploration of network connectivity of multi-site recorded data available in the close future. As a proof of concept, in this paper, we test and benchmark our approach on simulated data generated by a realistic network of cerebellar granular cells, for which the structural connectivity is known. The method we propose is based on the hypothesis that the network connectivity is sparse. Several theoretical, computational, and experimental studies [13] suggested 
that neurons encode sensory information using a small number of active neurons at any given point in time (this strategy, referred to as sparse coding). For example, sensory neurons fire in response to a very small fraction of all presented stimuli, and higher cortical neurons tend to fire relatively little in time. Moreover, an important theoretical result stated that if synapses are constrained to be either excitatory or inhibitory, then near capacity, the optimal solution is sparse [14] with most of the synapses silent. This result has been proposed as a functional explanation for the abundance of silent synapses in the cerebellum and other brain areas. We remark that sparse coding has been proposed as a guiding principle in neural representations of sensory input, particularly in the visual system. Because sparse codes are defined as representations with low activity ratios, i.e. at any given time a small proportion of neurons are active, they are sometimes proposed as a means to help conserve metabolic costs [15].

The structure of this paper is as follows. In Section 2 we present a general setting for the problem and we introduce a family of penalized regression techniques useful for identifying sparse effective connectivity patterns. In Section 3 we describe our experimental settings and data preprocessing. The results are reported in Section 4, while final remarks are postponed in the last section.

\section{A new Network reconstruction method}

\subsection{Problem Formulation}

Let us consider a graph $G=(V, E)$, where $V$ corresponds to a set of $N$ neurons connected with a directed graph given by the edges contained in $E$ of ordered pairs of vertices contained in $V \times V$. On each node $i \in V$, two stochastic processes are observed:

- an external process $\left\{X_{i}(t), 0 \leq t \leq T\right\}$ (spike);

- an internal process $\left\{Y_{i}(t), 0 \leq t \leq T\right\}$.

We assume a Local Markov Property (LMP): the relevant information on each process $Y_{i}(t), i=1, \ldots, N$ is "contained" on the external activity of the neurons $j$ connected to it, and in a time interval $\delta$. For example, in the network given in Fig. 1, the law of the 4th neuron depends on the external activity of the 1st, 2nd, and 5th neuron together with its own internal activity.

We explain now the LMP in terms of the $\sigma$-algebras of events observable by the processes. We denote by $\mathcal{F}_{t^{-}}$the observable events of the "past" of the external processes until time $t$, and it will be defined as the $\sigma$-algebra of events generated by the processes $\left\{X_{i}(s), s<t, i \in V\right\}: \mathcal{F}_{t^{-}}=\bigvee_{i \in V} \sigma\left(X_{i}(s), s<t\right)$. The $\sigma$-algebra $\mathcal{F}_{t^{-}}^{(i)}$ contains the observable events of the "close past" of the in-neighborhoods of the $i$-th neuron: $\mathcal{F}_{\delta, t^{-}}^{(i)}=\bigvee_{j:(j, i) \in E} \sigma\left(X_{j}(s), t-\delta \leq s<t\right)$. Moreover, we will denote by $\mathcal{G}_{t^{+}}$the observable events of the "future" of the internal processes: $\mathcal{G}_{t^{+}}^{(i)}=\sigma\left(Y_{i}(u), t \leq u\right)$.

With this notation, LMP says that the future of the $i$-th internal process and past of all the external processes are conditionally independent given the close past of the $i$-th neuron:

$$
P\left(G \mid \mathcal{F}_{t^{-}}\right)=P\left(G \mid \mathcal{F}_{\delta, t^{-}}^{(i)}\right), \quad \forall G \in \mathcal{G}_{t^{+}}^{(i)}, \forall i
$$

In other words, $\mathcal{F}_{\delta, t^{-}}^{(i)}$ gives all the relevant information on the future of the internal activity of the $i$-th neuron.

\subsection{Event-based discrete time model}

We use a particular model of the class of processes satisfying (LMP). We sample the processes at discrete times $t_{m}=m \delta$, and we assume an event-based model: both the internal and external processes are simple point processes, and we assume that at most one event may occur for each process in any time interval. We note that the definition of event depends on the knowledge of the investigator and on the available data. For the purpose of this paper, the definition of events will be introduced in the Section 3.2. 


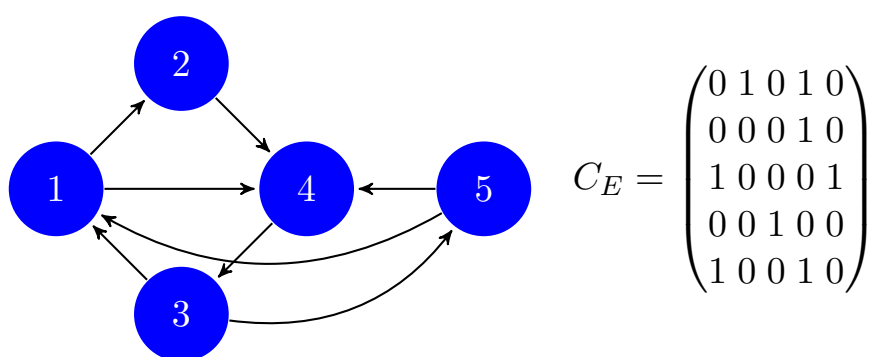

Figure 1. Example of a nework of 5 neurons and its adjacency matrix.

Given an event-based model, for any $m=1, \ldots, M$, we observe

$$
y_{i}^{m}= \begin{cases}+1 & \text { if there has been an event for } Y_{i}(s) \text { during }\left(t_{m-1}, t_{m}\right] ; \\ 0 & \text { otherwise; }\end{cases}
$$

and

$$
x_{i}^{m}= \begin{cases}+1 & \text { if there has been an event for } X_{i}(s) \text { during }\left(t_{m-1}, t_{m}\right] \\ 0 & \text { otherwise; }\end{cases}
$$

The model can be completely characterized by defining the family of conditional probabilities: for any $i, m$,

$$
\pi(i, m)=P\left(y_{i}^{m+1}=1 \mid x_{j}^{l}, l \leq m, j \in V\right)=P\left(y_{i}^{m+1}=1 \mid x_{j}^{m},(j, i) \in E\right)
$$

the last equality being a consequence of (LMP).

To model the association among the nodes, we assume in this paper a time-homogeneous linear logit model:

$$
\pi(i, m)=\left(1+\exp \left(-\beta_{0}-\sum_{(j, i) \in E} \beta_{j, i} x_{j}^{m}\right)\right)^{-1}
$$

Given a set $\left\{\omega_{i, m}\right\}$ of positive real numbers, the negative weighted log-likelihood function for our processes reads:

$$
\ell_{M}(\boldsymbol{\beta})=-\sum_{m=1}^{M-1} \sum_{i \in V} \omega_{i, m}\left(y_{i}^{m+1} \log \pi(i, m)+\left(1-y_{i}^{m+1}\right) \log (1-\pi(i, m))\right)
$$

Note that the significant parameters $\left\{\beta_{j, i},(j, i) \in E\right\}$ of our model depend on the topology of the network. The choice of the weights is done here to balance the number of $y_{i}^{m}=0$ and $y_{i}^{m}=1$. A simple choice might be $\omega_{i, m}=\sum_{j, l} y_{j}^{l}$ if $y_{i}^{m}=0$ and $\omega_{i, m}=\sum_{j, l}\left(1-y_{j}^{l}\right)$ if $y_{i}^{m}=1$.

\section{From Data to links}

In general, we must follow three steps (see Fig. 2): signal acquisition, signal filtering and detection of the events of interest, and reconstruction of the network connections. This work focuses essentially on the last step but, in the following, we will also give some details about the model used to simulate the data and the pre-processing of these data. The filtering algorithm will be described in a forthcoming work [16] and it is based on a modified non local, non linear anisotropic diffusion method. This algorithm has been already used in [17].

\subsection{Cerebellar Simulated data}

We simulate the potential of the $j$-th neuron $V_{j}(t)$ by the Hodgkin\&Huxley equations of the cerebellar granule cell (GrC, [18]). Heterogeneity among the GrCs is introduced by randomizing the resting potentials with a current of $2 \pm 0.2 \mathrm{pA}$. The network consists of 20 neurons with a connectivity probability 


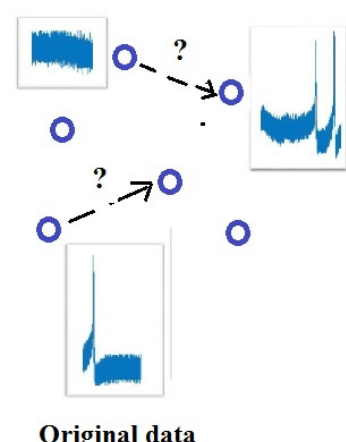

(A)

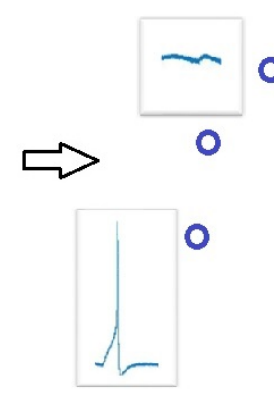

Filtered signals

(B)

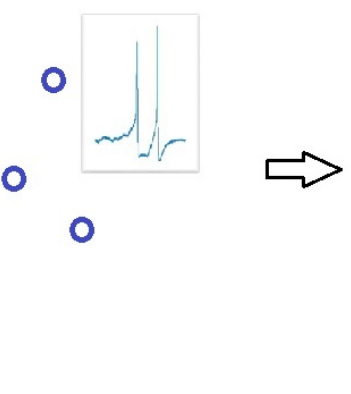

)

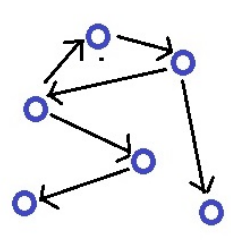

Recovered links

(C)

Figure 2. The flow chart shows the three main steps for identifying the connections of a neural network: (A) the acquisition of the original signal, with some noise; (B) the filtered signal (with the detection of the events for the reconstruction); (C) the subsequent penalized regression techniques useful for identifying sparse effective connectivity patterns.

of 0.3 and all connections are directional (i.e. chemical synapses). Since self-connections (i.e. 'autapses') are not so frequent in the brain, they were not included in our network. Based on these numbers, the simulated network comprises on average 114 connections. The chemical synapses are modeled by the fast AMPA excitatory currents [19]. In addition, to mimic a biologically realistic noisy regime, Poisson distributed AMPA currents (of frequency $0.2 \mathrm{~Hz}$ ) are injected to all GrCs. The cellular and synaptic models are described in the literature $[18,19]$ and the parameter settings and their changes respect to the literature are reported in the Table 1. Since here we are interested in highlighting the potential impact

\begin{tabular}{lllll}
\hline synaptic input & parameter & unit & value & value in literature [19] \\
AMPA & gmax & $\mathrm{pS}$ & 800 & 1200 \\
& $r_{1}$ & $m s^{-1} m M^{-1}$ & 5.4 & \\
& $r_{2}$ & $m s^{-1}$ & 0.84 & \\
& $r_{6}$ & $m s^{-1} m M^{-1}$ & 0 & 1.12 \\
NMDA & not included here & & & \\
synaptic noise & gmax & & & \\
& $r_{1}$ & $m s^{-1} m M^{-1}$ & 5.4 & \\
& $r_{2}$ & $m s^{-1}$ & 0.1 & \\
& $r_{6}$ & $m s^{-1} m M^{-1}$ & 0 \\
\hline
\end{tabular}

of our approach we discarded the contribution of other synaptic conductances that will be included in a next work.

We simulate five seconds of activity of such a network. A snapshot of the activity of a neuron in the network is shown in Fig. 3. The upswings of the potential are mainly determined by the inputs from the other cells (the time occurrence of these inputs is highlighted by the red, blue and green dashed lines). Interestingly, the spiking activity is not strictly determined by the input itself. In fact, the spike doublet $(\mathrm{D} 1, \mathrm{D} 2)$, the isolated spikes (IS) and the spike from excitation (EXC) are not apparently determined by the input. Spike D1 arises from a depolarization that is not directly caused by the input and spike D2 can either by determined by high membrane excitation as well as by noise. The spike IS is most probably caused only by noise and spike EXC is again due to membrane excitation or simply by synaptic noise. In other words, we can simulate a network activity starting from the biophysical models of a single cell or synapse. In this work these are the test data. 


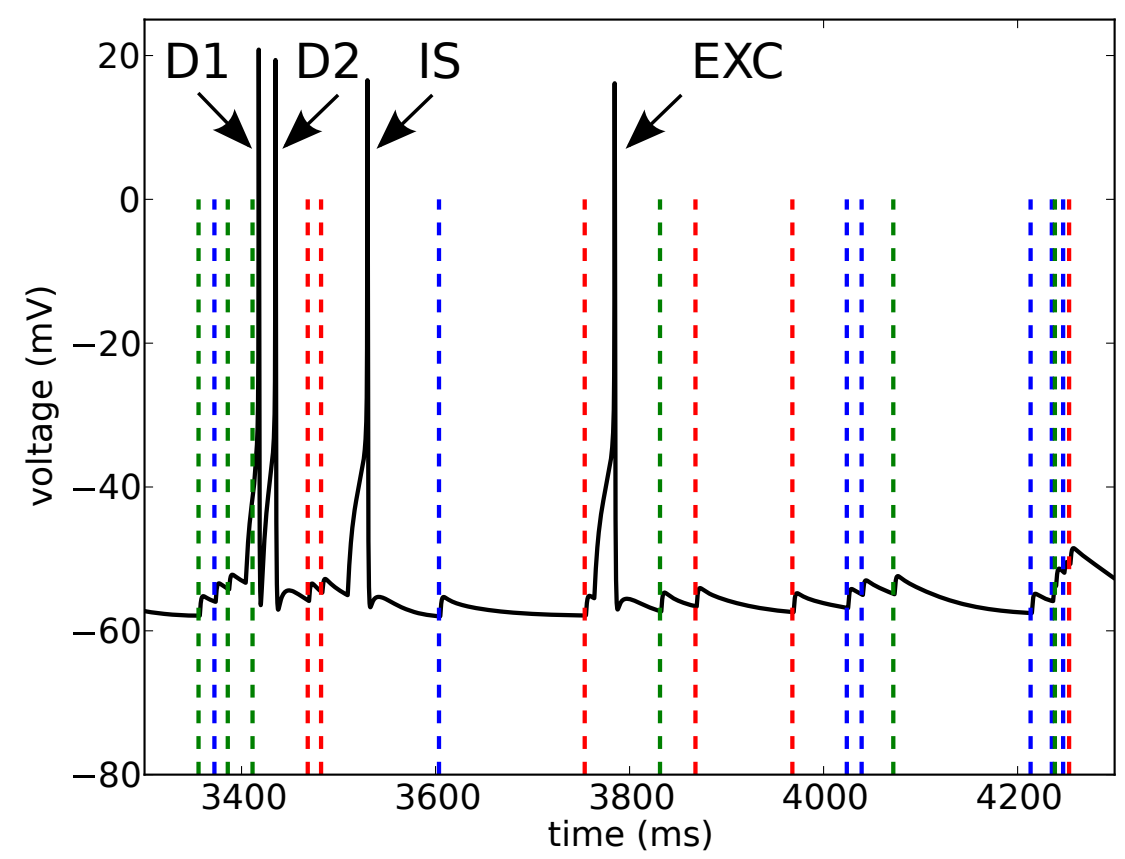

Figure 3. Voltage trace of a neuron in the network. The voltage (black trace) is overlayed with the time stamps of the inputs (colored dash lines) to the cell. The voltage changes are clearly correlated with the input. However spikes D1, D2, IS, EXC are not apparently determined solely by the input. Note that the blue, red and green colors correspond to three different cells.

\subsection{Data preprocessing}

The process $\left\{x_{j}^{m}, m=1, \ldots, M\right\}$ is the discretization (in time) of the spike activity of the $j$-th neuron, so that $x_{j}^{m}=1$ if there has been a spike during the time interval $\left(t_{m-1}, t_{m}\right]$.

The process $\left\{y_{j}^{m}, m=1, \ldots, M\right\}$ is a reaction process, nonlinear filter of the potential activity $V_{j}(t)$. Here, $y_{j}^{m}=1$ if there has been an event for $V_{j}(t)$ during the time interval $\left(t_{m-1}, t_{m}\right]$, where an event at time $t$ depends on three factors (see Fig. 4):

i. the right derivative $V_{j}^{\prime}\left(t^{+}\right)$must be greater than a positive threshold (increase of potential after excitation);

ii. the increase of derivatives $V_{j}^{\prime}\left(t^{+}\right)-V_{j}^{\prime}\left(t^{-}\right)$must be greater than a positive threshold (convex effect of potential due to excitation);

iii. the left derivative $V_{j}^{\prime}\left(t^{-}\right)$must be greater than a negative threshold (in connection with other conditions, this avoids an event caused by the recovery of the resting potential following the hyperpolarization phase; alone, this identifies those events).

More precisely, when a sequence of times of length $n \geq 1$ satisfies the requested conditions, an event is detected. The first time of this sequence is the time of the event.

\subsection{Topology estimator}

Given the data of our processes, we are interested in reconstructing the topology of our network. We want to give an estimator $\hat{V}$ of the set $V$ of edges. In our contest, the edge $(j, i)$ exists when the process $y_{i}^{m}$ is directly caused by $x_{j}^{m}$, in the sense of (LMP). In other words, when $\beta_{j, i}$ is different from zero in (2). Therefore, we adopt the following strategy:

- with a penalization technique, we find a sparse estimator $\hat{\boldsymbol{\beta}}$ of $\boldsymbol{\beta}$;

- we say that $(j, i) \in \hat{V}$ if $\hat{\beta}_{j, i}$ is different from zero. 

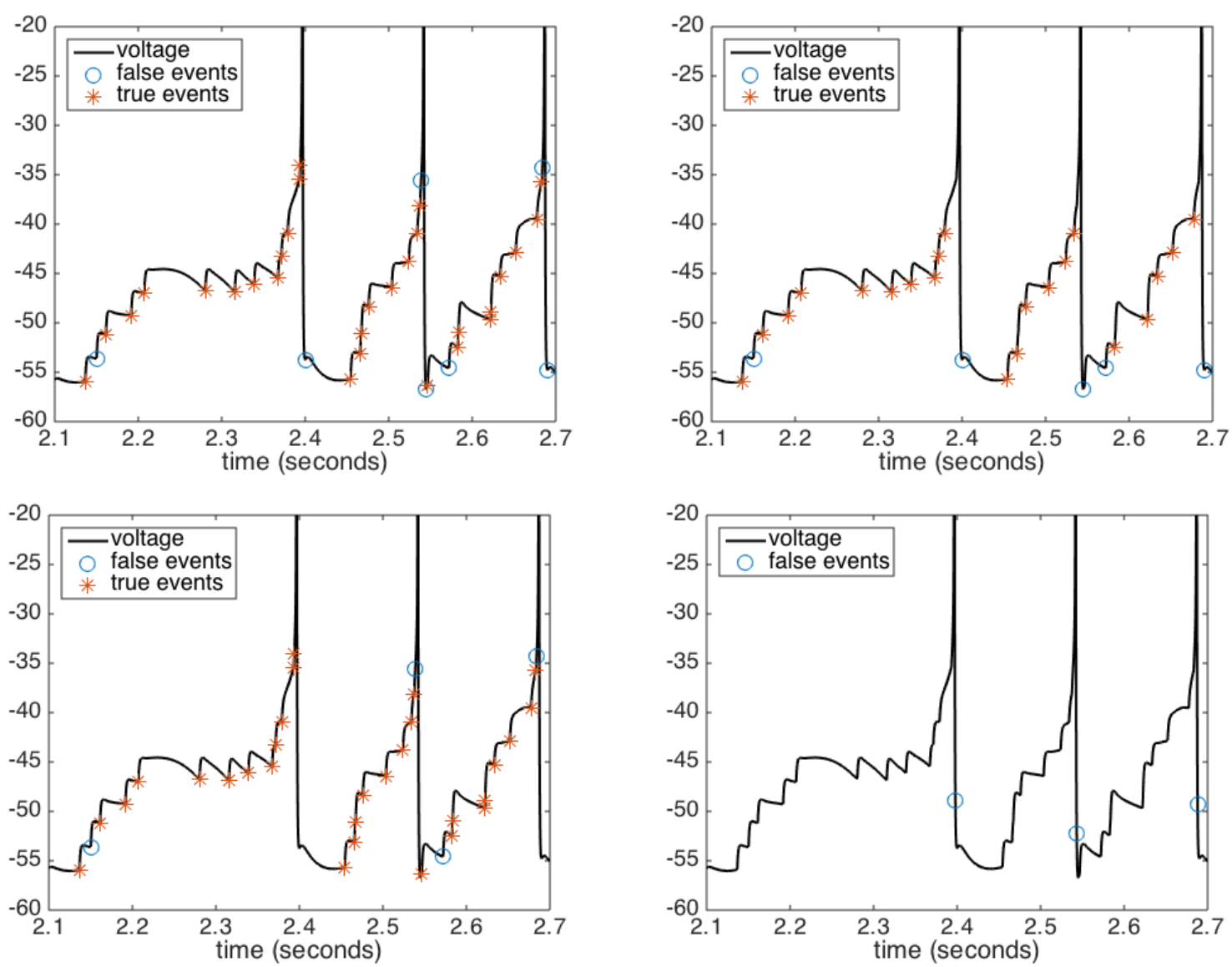

Figure 4. Example of identification of events based on the voltage activity. Events that are determined by the links of the network are marked with $*$ (i.e. true positive), while $\circ$ shows false identification (i.e. false positive) (due, for example, to external noise). Top left: identification of events with all the three factors of data preprocessing. Top right: identification of events with only (i) factor. Bottom left: identification of events with only (ii) factor. Bottom right: identification of events with only (iii) factor.

In this paper, we adopt a $\ell_{1}$-penalization on the regression coefficients $\left\{\beta_{j, i}, i, j \in E\right\}$. Given a positive penalization parameter $\lambda$, let

$$
L(\boldsymbol{\beta}, \lambda)=\ell_{M}(\boldsymbol{\beta})+\lambda \sum_{i, j \in E}\left|\beta_{j, i}\right|
$$

and define the Lasso estimator $\hat{\boldsymbol{\beta}}(\lambda)=\arg \min _{\boldsymbol{\beta}} L(\boldsymbol{\beta}, \lambda)$. As stated above, we define $\hat{V}(\lambda)$ as

$$
(j, i) \in \hat{V} \Longleftrightarrow \hat{\beta}_{j, i}(\lambda)>0 .
$$

Here we also compare the performances of the novel methodology with the standard cross-correlation that is widely used in the multi electrode array field [20-22]. The cross-correlation functions among the discrete spike trains are defined as:

$$
C C_{i, j}(\tau)=\frac{\left\langle S T_{i} \cdot S T_{j}(\tau)\right\rangle}{\sqrt{N_{i} \cdot N_{j}}}
$$

where $S T_{i}, S T_{j}$ are the binned spike trains (bin size $1 \mathrm{~ms}$ ) and $N_{i}, N_{j}$ the corresponding number of spikes. The strength of a connection, between nodes $i$ and $j$, is given by the peak of the quantity $C C_{i, j}$ of Eq. (5). The network topology can then be inferred by retaining the strongest and most significants cross-correlation peaks that are overcome a selected threshold. 


\section{Experimental Results}

The validation of the proposed methodology is afforded on simulations presented in the Section 3.1. The performances of the methodologies are quantified with the well known receiver operating characteristic (ROC) curves and with the recently introduced positive precision curve (PPC, [20]). The graphs reported in Fig. 5 are obtained by varying the penalization parameter $\lambda$, for the Lasso methodology, and the cross-correlation threshold, for the respective correlation methodology.

Let us remind that PPC is defined as:

$$
\mathrm{PPC}=\frac{T P-F P}{T P+F P}
$$

and represents the proportion of the correctly (true positive, TP) versus the incorrectly (false positive, FP) inferred links. The plot on the right in the Fig. 5 reports the PPC index with respect to the number of links included in the analysis. Interestingly, the PPC curve of the Lasso stays at its maximum up to $30 \%$ of the links, which corresponds to the real links of the network (the connectivity probability is 0.3 ). Clearly, beyond $30 \%$ of the included links, the PPC has a negative power decay. We point out that the cross-correlation does not achieve to infer the topology at any level of the cross-correlation threshold. Interestingly, when the Lasso events are based only on spiking information (condition (iii) alone) it performs as bad as the cross-correlation. The Lasso (Fig. 5) performs very well and reaches the maximum value $(=1)$ in both the ROC and PPC curves for a large range of the penalization parameter. Moreover, the worst performances are achieved when no constraint is imposed on the sparsity (e.g. $\lambda=0$ ) of the inferred network. Finally, the intuition is preserved: the first connections introduced in the Lasso estimate, are almost all true (PPC 1). Another interesting feature of the newly introduced Lasso methodology consists into its capability of inferring the bidirectional links. The results of Fig. 5 are based on a network with 121 connections out of which 38 were bidirectional ( $31.4 \%$ out of the total). This is another advantage over the cross-correlation method, which only determines unidirectional connections.
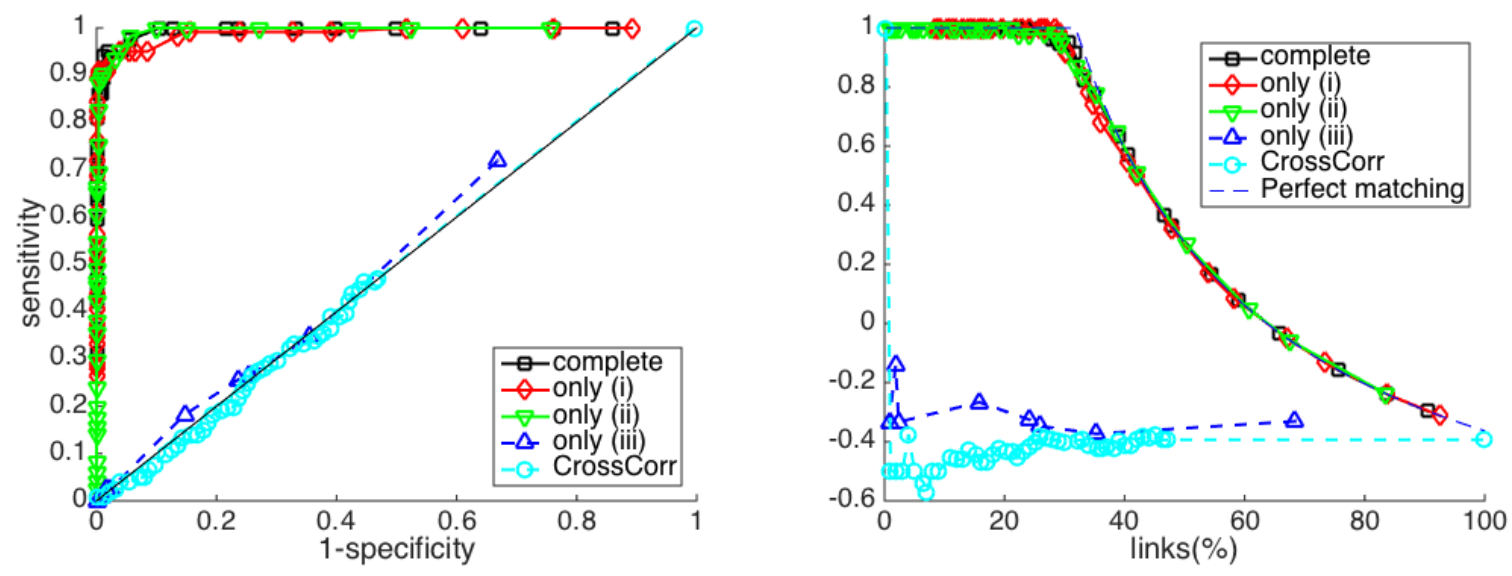

Figure 5. ROC (on the left) and PPC (on the right) curves for the topology estimator (4), with the complete data preprocessing and with each of the filtering conditions (i), (ii) and (iii) given in Section 3.2. The first conditions (i) and (ii) of data preprocessing may be used alone without affecting the main result, while spiking activity alone is not predictive

\section{Conclusions and Future Works}

Understanding how interactions between brain structures support the performance of specific cognitive tasks or perceptual processes is a prominent goal in neuroscience. The effects that one part of the nervous system has on another are typically examined by stimulating or lesioning the first part and investigating the outcome in the second. For example, in peripheral and spinal pathways, the interventional techniques of stimulation and ablation have proven to be powerful methods for inferring causal influences from one 
neuron or neuronal population to another. For the study of causal relations within the brain (functional and effective connectivity), the utility of the interventional techniques is diminished by the high levels of convergence and divergence in brain pathways.

In this work, we have developed an event-based approach for inferring networks of causal relationships in a neuronal population. Specifically, we suppose that we are able to observe the dynamical behaviors of individual components of a neural network and that few of the components may be causally influencing each other. The variables could be invasive electrode recordings, intracranial EEG, or non-invasive EEG, MEG or fMRI time series from different parts of the brain. In order to introduce our method, we have considered a simulated cerebellar granule cell network that captured the nonlinear interactions even in both short and noisy time series.

The results we got are quite promising from many points of view. First, despite the algorithm was applied only to short simulations ( 5 seconds) it achieved to filter out noisy from causal responses yielding a reliable estimate of the underlying connections in the network. Second, the approach is quite general and the conditions (i),(ii),(iii) can be further adapted to different types of electro-physiological signals. Third, the Lasso is also quite robust respect to bidirectional connections in the network. This is of fundamental importance since bidirectional network motifs are quite abundant in the brain [23]. The proposed Lasso methodology assumes the knowledge of the voltage traces, regardless of the particular properties of the neurons of a given network. From an experimental point of view, such a detailed information can be achieved with patch-clamp experiments, but this technique is not designed to perform simultaneous recordings from populations of neurons. Interestingly, nowadays there have been enormous improvements in the field of the genetically encoded voltage indicators that allow tracking sub-threshold activities [24], a key ingredient of the Lasso algorithm. This progress will likely provide, in the near future, multi-site recordings of a population of neurons.

As a next step, we will then test the robustness of the methodology including different noise sources (i.e. membrane noise), inhibitory connections and verify its robustness respect to bigger networks.

\section{References}

1. I. Stevenson and K. Kording, How advances in neural recording affect data analysis., Nat Neurosci, vol. 14, pp. 139-142, Feb 2011.

2. M. Churchland, B. Yu, M. Sahani, and S. K.V., Techniques for extracting single-trial activity patterns from large-scale neural recordings., Curr Opin Neurobiol, vol. 17, no. 5, pp. 609-618, 2007.

3. E. Bullmore and O. Sporns, Complex brain networks: Graph theoretical analysis of structural and functional systems, Nature Reviews Neuroscience, vol. 10, no. 3, pp. 186-198, 2009.

4. A. Bolstad, B. D. Van Veen, and R. Nowak, Causal network inference via group sparse regularization, IEEE Transactions on Signal Processing, vol. 59, no. 6, pp. 2628-2641, 2011.

5. M. Winterhalder, B. Schelter, W. Hesse, K. Schwab, L. Leistritz, D. Klan, R. Bauer, J. Timmer, and W. H., Comparisson of linear signal processing techniques to infer directed interactions in multivariate neural systems., Signal Process., vol. 85, no. 11, pp. 2137-160, 2005.

6. C. Granger, Investigating causal relations by econometric models and cross-spectral methods., Econometrica, vol. 37, pp. 424-438, 1969.

7. S. Bressler and K. Anil, Granger causality: A well established methodology., NeuroImage, vol. 58, no. 2, pp. 323-29, 2011.

8. A. Brovelli, D. Mingzhou, A. Ledberg, Y. Chen, R. Nakamura, and B. S.L., Beta oscillations in a largescale sensorimotor cortical network: Directional influences revealed by Granger causality, Proceedings of the National Academy of Sciences of the United States of America, vol. 101, no. 26, pp. 9849-9854, 2004.

9. S. Kim, D. Putrino, S. Ghosh, and E. Brown, A Granger Causality Measure for Point Process Models of Ensemble Neural Spiking Activity, PLOS Computational Biology, vol. 7, pp. 1-13, 032011.

10. A. Cadotte, T. DeMarse, P. He, and M. Ding, Causal measures of structure and plasticity in simulated and living neural networks, PLOS ONE, vol. 3, pp. 1-14, 102008. 
11. R. Vardi, A. Goldental, S. Sardi, A. Sheinin, and I. Kanter, Simultaneous multi-patch-clamp and extracellular-array recordings: Single neuron reflects network activity., Scientific Reports., vol. 6, p. 36228, 2016.

12. K. Deisseroth, Optogenetics: 10 years of microbial opsins in neuroscience., Nature neuroscience, vol. 18, no. 9, pp. 1213-1225, 2015.

13. B. Olshausen and F. D.J., Sparse coding of sensory inputs., Current Opinion in Neurobiology, vol. 14, pp. 481-487, 2004.

14. N. Brunel, V. Hakim, P. Isope, J. Nadal, and B. B., Optimal information storage and the distribution of synaptic weights: perceptron versus purkinje cell., Neuron, vol. 43, no. 5, pp. 745-57, 2004.

15. E. Bullmore and O. Sporns, The economy of brain network organization, Nature Reviews Neuroscience, vol. 13, no. 5, pp. 336-349, 2012.

16. G. Aletti, M. Moroni, and G. Naldi, A new nonlocal nonlinear diffusion equation for image denoising and data analysis, arXiv: 1707.06396, 2017.

17. G. Palazzolo, M. Moroni, A. Soloperto, G. Aletti, G. Naldi, M. Vassalli, T. Nieus, and F. Difato, Fast wide-volume functional imaging of engineered in vitro brain tissues, Scientific Reports, vol. 7, no. 1, 2017.

18. E. D'Angelo, T. Nieus, A. Maffei, S. Armano, P. Rossi, V. Taglietti, A. Fontana, and N. G., Thetafrequency bursting and resonance in cerebellar granule cells: Experimental evidence and modeling of a slow k+-dependent mechanism, Journal of Neuroscience, vol. 21, no. 3, pp. 759-770, 2001.

19. T. Nieus, E. Sola, J. Mapelli, E. Saftenku, P. Rossi, and D. E., Ltp regulates burst initiation and frequency at mossy fiber-granule cell synapses of rat cerebellum: experimental observations and theoretical predictions., J Neurophysiol, vol. 95, pp. 686-699, Feb 2006.

20. M. Garofalo, T. Nieus, P. Massobrio, and M. S., Evaluation of the performance of information theorybased methods and cross-correlation to estimate the functional connectivity in cortical networks., PLoS One, vol. 4, no. 8, p. e6482, 2009.

21. A. Maccione, M. Garofalo, T. Nieus, M. Tedesco, L. Berdondini, and S. Martinoia, Multiscale functional connectivity estimation on low-density neuronal cultures recorded by high-density cmos micro electrode arrays., J Neurosci Methods, vol. 207, pp. 161-171, Jun 2012.

22. S. Ullo, T. Nieus, D. Sona, A. Maccione, L. Berdondini, and M. V., Functional connectivity estimation over large networks at cellular resolution based on electrophysiological recordings and structural prior., Front Neuroanat, vol. 8, p. 137, 2014.

23. S. Song, P. Sjostrom, M. Reigl, S. Nelson, and C. D.B., Highly nonrandom features of synaptic connectivity in local cortical circuits., PLoS Biol, vol. 3, p. e68, Mar 2005.

24. Y. Gong, C. Huang, J. Li, Z. Jin, B. Grewe, Y. Zhang, S. Eismann, and S. M., High-speed recording of neural spikes in awake mice and flies with a fluorescent voltage sensor., Science (New York, N.Y.), vol. 350, pp. 1361-1366, Dec 2015. 